CAHIERS

MONDES

ANCIENS

\section{Cahiers « Mondes anciens »}

Histoire et anthropologie des mondes anciens

$13 \mid 2020$

Qu'est-ce que faire école ? Regards sur « l'école de

Paris »

\title{
Par delà les frontières ?
}

Beyond the Borders?

\section{Paul Demont}

\section{(2) OpenEdition}

Journals

Édition électronique

URL : http://journals.openedition.org/mondesanciens/2566

DOI : 10.4000/mondesanciens.2566

ISSN : 2107-0199

Éditeur

UMR 8210 Anthropologie et Histoire des Mondes Antiques

Référence électronique

Paul Demont, «Par delà les frontières ? », Cahiers " Mondes anciens » [En ligne], 13 | 2020, mis en ligne le 10 juin 2020, consulté le 17 juin 2020. URL : http://journals.openedition.org/mondesanciens/2566 ; DOI : https://doi.org/10.4000/mondesanciens.2566

Ce document a été généré automatiquement le 17 juin 2020.

\section{(c) $($ ) $(9)$}

Les Cahiers «Mondes Anciens » sont mis à disposition selon les termes de la licence Creative Commons Attribution - Pas d'Utilisation Commerciale - Pas de Modification 4.0 International. 


\title{
Par delà les frontières ?
}

\author{
Beyond the Borders?
}

\section{Paul Demont}

Commençons par une question de terminologie, qui a son importance. L'appellation «École de Paris " n'est venue à ma connaissance que relativement récemment, par l'intermédiaire de son usage aux Amériques. En Europe, et a fortiori en France, j'ai toujours rencontré des individus plus qu'une école.

2 Remontons donc à ces premières rencontres, dans les années 1970, celles d'un jeune étudiant en «Lettres classiques » (une spécialité qui, en France, est distincte des études d'histoire et de philosophie, et qui associe le latin et le grec à l'étude de la littérature française), né en 1949, élève à l'École normale supérieure de 1969 à 1972. Je crois bien avoir découvert les nouveautés introduites par ces individualités alors atypiques qui commençaient à constituer un groupe autour de Jean-Pierre Vernant, à l'École normale supérieure, à l'occasion d'une intervention de François Hartog sur ce qui allait devenir Le miroir d'Hérodote. De ce groupe, il n'avait quasiment pas été question auparavant, me semble-t-il, dans mes études de « khâgne » (autre spécialité française : les deux ou trois années après le baccalauréat pendant lesquelles un étudiant prépare, en dehors de l'université, dans les « classes préparatoires » des lycées, les « concours » d'entrée dans les «grandes écoles »). Le «bœuf autocuiseur » des Scythes, et l'étude du jeu avec la conception supposée "grecque " du sacrifice, comblèrent d'aise les auditeurs, dont j'étais. Plus tard, quand je fus très provisoirement (pour un an), assistant de Jacqueline de Romilly (qui dirigeait ma thèse) à la Sorbonne, pour remplacer Suzanne Saïd (qui était en séjour d'un an aux États-Unis), je suivais à la fois les séminaires de Jacqueline de Romilly, de Louis Robert (au Collège de France), de Jean Irigoin (son séminaire de métrique à l'École normale supérieure), de Jean Bollack (un séminaire dont il avait conquis de haute lutte l'existence à l'École normale supérieure, avec l'aide de Jean Lallot) et de Jean-Pierre Vernant (à l'École Pratique des Hautes Études), ainsi que le séminaire de patristique du tout jeune Alain Le Boulluec à l'École normale supérieure, qui était " caïman » à l'ENS : six façons extrêmement différentes d'aborder l'antiquité grecque (il y en avait d'autres encore à Paris, bien sûr, mais je les fréquentais moins). Il 
fallait une certaine tendance à la schizophrénie pour passer de l'un à l'autre, mais je ne regrette pas cette gymnastique intellectuelle.

3 Le séminaire de Vernant me séduisait en particulier par la présentation, très fouillée, des ouvrages récents qu'il avait reçus, et qui venaient le plus souvent d'auteurs proches de sa méthode, et très souvent présents dans la salle de l'École Pratique des Hautes Études (EPHE) où il enseignait alors : il les lisait et il les interprétait longuement, très longuement, devant ses auditeurs avec une magnifique empathie. Cette empathie si remarquable n'est certainement pas, à mes yeux, l'un des moindres éléments qui expliquent la constitution de son groupe, sinon de son école, et la solidité de ce groupe. Il était le seul des maitres à tutoyer ses auditeurs, lesquels, souvent, continuaient de le vouvoyer, ce qui introduisait une curieuse déformation de la relation qu'il souhaitait. Désireux d'impliquer dans son séminaire (voire peut-être avec l'arrière-pensée de " mouiller ») un petit jeune de la Sorbonne (dont il était exclu), il m'avait proposé d'y faire un exposé, ce que j'avais accepté avec enthousiasme et inquiétude. J'étais à l'époque très intéressé par les travaux de ce qu'on appelait «l'école» structuraliste (une autre "école» aux cent têtes), et j'avais tenté d'utiliser les travaux de Claude Brémond pour analyser, d'un point de vue narratologique, un passage d'Hérodote. Je me souviens de cette séance et je revois encore Pierre Vidal-Naquet et Marcel Detienne, assis côte à côte, ne cessant de bavarder comme des collégiens pendant cet exposé, qui était resté très classique, voire traditionnel, en fait (mais qui a failli ruiner ma future carrière universitaire à la Sorbonne : un exposé chez Vernant !).

4 Revenu, malgré cet écart de conduite, en 1975, à la Sorbonne comme "assistant» titulaire de mon poste cette fois (car une telle situation, si favorable pour un jeune doctorant, a bel et bien existé), j'y ai mieux connu mes collègues, et notamment «le cadre B» (c'est-à-dire les enseignants qui n'avaient pas, ou pas encore, le rang de " professeur ») : Suzanne Saïd, elle aussi disciple fidèle de Jacqueline de Romilly, mais très proche des travaux de Jean-Pierre Vernant et de Pierre Vidal-Naquet sur la tragédie - pour elle comme pour moi, la notion d'une «école de Paris » autour d'eux aurait paru étrange, à ce moment-là, je crois. J'étais aussi collègue de Jean-Louis Durand, qui était lui aussi "assistant", et avec qui j'eus des discussions passionnées autour du structuralisme, mais cette fois dans la version de Greimas, à l'époque où Jean-Louis élaborait et réélaborait avec Vernant l'analyse des récits hésiodiques sur le sacrifice : il était, lui, beaucoup plus nettement de "l'école ", avec son intérêt si vif et si compétent pour l'anthropologie. Je nous revois encore noircissant tous deux le tableau de schémas narratologiques autour du mythe de Prométhée. Ce fut peut-être mon contact le plus approfondi avec le groupe.

Dans ces années, avaient été introduites à la Sorbonne (horresco referens), pour des étudiants de Lettres qui étaient de moins en moins capables, à leur arrivée à l'université, de lire les textes en grec, des unités d'enseignement sur textes traduits : Jean-Louis et moi en étions chargés pour le grec; je fis alors la connaissance de Florence Dupont, qui enseignait alors aussi à la Sorbonne et en était responsable pour la littérature latine. C'est par leur intermédiaire et grâce à leur enthousiasme que je pris le mieux conscience de la nécessité et de la possibilité de l'adaptation de l'enseignement traditionnel, et aussi des conséquences que devaient avoir les nouvelles méthodes (et parfois les nouveaux dogmes) de lecture des textes sur l'enseignement lui-même. De là date une amitié solide avec Florence (rencontrée auparavant à Aussois, dans le séminaire annuel fondé par Jean Lallot, qui n'a pas, lui, conduit à une «école ", 
mais a été important pour beaucoup), malgré les différences d'orientation et les réserves que pouvaient susciter chez moi certains aspects de ses travaux sur l'épopée et la tragédie grecques. J'assurais aussi, bien sûr, et surtout, des cours sur textes grecs, et je voudrais ici évoquer aussi la mémoire d'une amie très chère, Annie (ou Anne) Lebeau, qui savait mieux que personne allier l'exigence philologique à la rigueur de l'analyse littéraire: les séminaires que nous avons donnés en commun sur le théâtre grec antique, alternativement l'un et l'autre, mais chacun assistant au cours de l'autre et en discutant après la séance, restent l'un de mes plus beaux souvenirs d'enseignant. Les travaux de « l'école de Paris » (mais aussi ceux de Froma Zeitlin ou de Charles Segal, par exemple, qui ne sont pas spécialement "parisiens») y trouvaient leur place, parmi beaucoup d'autres, et nous ont aidés à proposer à nos étudiants des approches une peu différentes de ce que nous avions reçu de nos maîtres.

Un dernier souvenir de ces années de jeunesse : je revois encore François Lissarrague venir régulièrement voir Jean-Jacques Maffre pour acquérir cette culture de céramologue classique qu'il ne possédait pas, que Jean-Jacques lui a si libéralement ouverte et dont il a fait un si bel usage.

7 Mon sujet de thèse avait été choisi avec Jacqueline de Romilly dans le cadre de "l'histoire des idées", mais c'est plutôt dans la perspective des recherches contemporaines sur la sémantique (notamment celles de John Lyons et de John Searle) que j'ai commencé mes propres travaux de recherche - car j'ai toujours pensé que l'histoire des idées, la sémantique et l'analyse de la communication entre les humains étaient indissociablement liées. Mon premier article, à propos des recherches

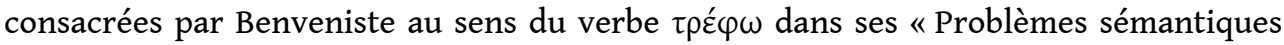
de la reconstruction ", a bénéficié de conseils très précieux de Jean Irigoin, Jacques Jouanna et Jean Taillardat, et a été bien accueilli par Vernant et ses amis en raison des implications qu'il avait pour l'anthropologie de la Grèce antique, mais c'était le travail d'un philologue extérieur au groupe, qui découvrait alors, grâce à Jacques Jouanna, la médecine hippocratique. Parallèlement, je poursuivais ma thèse sur La cité grecque archaïque et classique et l'idéal de tranquillité, toujours entre histoire des idées et sémantique, sans jamais exposer quoi que ce soit de ces travaux, où que ce soit (sauf devant des philosophes - un grand merci à Pierre Aubenque -, intéressés par les soubassements sémantiques et culturels des idéaux de $\sigma \chi \chi 0 \lambda \eta ́)$. Quand elle eut été soutenue, j'ai demandé à plusieurs amis et connaissances de m'aider, après les remarques du jury, à la corriger pour la publication. Nicole Loraux, entre autres, accepta de la relire et me dit avoir apprécié la première partie, et moins les parties suivantes (un jugement exactement inverse de celui de Jacqueline de Romilly...) : j'y avais en effet étudié la huitième Pythique de Pindare en m'efforçant d'adapter pour cela, le plus discrètement possible (car l'emploi d'un vocabulaire d'école m'est tout à fait étranger), les instruments de la narratologie, voire de l'anthropologie - dans une étude qui est restée largement inconnue des spécialistes de Pindare d'ailleurs. Cette requête venant de moi est peut-être l'une des raisons pour lesquelles elle m'a invité ultérieurement à plusieurs jurys de thèses qu'elle avait dirigées, ce dont je reparlerai.

8 L'influence grandissante exercée sur moi par le séminaire hippocratique de Jacques Jouanna et les relations de confiance ainsi établies entre lui et moi m'ont peu à peu conduit, à sa demande, à prendre des responsabilités dans l'Association des Études grecques, éditrice de la Revue des Études grecques, que dirigeait alors François Chamoux. Je lui ai finalement succédé comme Secrétaire général de cette association, par un 
hasard du calendrier, en juin 1987, l'année où Jean-Pierre Vernant en était le Président (la règle veut en effet dans cette association que la Présidence soit exercée chaque année par une personnalité différente). Il avait accepté d'assumer cette charge, qui témoignait de la reconnaissance du monde des hellénistes français pour son œuvre, malgré les antagonismes qui restaient vifs entre les philologues traditionnels et son " école ». C'est donc lui qui a présidé à mon élection. Il vaut peut-être encore la peine de mentionner quelques mots de la conclusion de son allocution de fin d'année, où il insistait sur la rupture que nous étions en train de vivre et qui est la principale source, à mon avis, des conflits entre hellénistes, et sur la nécessité de la «cohabitation » au sein de notre «tribu » (REG 100, 1987, p. XXV-XXXI) : «L'hellénisme avait sa place dans une culture générale qui l'avait, à sa façon, intégré et qui portait sa marque. C'est fini et ne reviendra pas. » J'ai fait ce que j'ai pu par la suite (je suis resté Secrétaire général jusqu'en 2002 et ai eu ensuite l'honneur de présider l'Association en 2004/2005) pour limiter ces conflits et pour défendre l'existence d'une communauté de recherche incluant les représentants des différentes écoles de pensée, car j'estime que, autant il est naturel d'organiser institutionnellement, comme cela a été fait avec méthode et efficacité, chaque école, autant il est dommageable pour tous que chacun fonctionne et se reproduise en vase clos.

9 Une nouvelle étape, pour moi, date de mon élection comme Professeur à l'université d'Amiens, une petite université dans laquelle la vie des études relatives à l'antiquité était déjà sans cesse sur le fil du rasoir. J'ai pu y inviter Jacqueline de Romilly, Jacques Jouanna, et plus tard, avec Pauline Schmitt Pantel, inviter aussi Jean-Pierre Vernant, profiter des relations d'amitié établies par Danièle Aubriot avec nos collègues de Liège (et faire ainsi la connaissance de Vinciane Pirenne-Delforge). Un grand moment fut la mise en scène de la traduction de la Phèdre de Sénèque par Florence Dupont, à mon initiative, qui déchaîna les passions amiénoises (mais que l'on n'a pas trop osé me reprocher, tant le succès éteignait les critiques) : pouvait-on, devait-on moderniser Sénèque à ce point? Tandis que Pauline orientait les travaux des historiens vers l'anthropologie et vers les études de genre (tout en insistant sur la composante philologique du travail historique : elle a eu la gentillesse de m'inviter à faire un cours et un séminaire historiques, et, plusieurs fois, à des jurys de thèse - et moi, je n'ai jamais « réciproqué ", à ma grande honte !), je m'efforçais d'ouvrir la philologie vers les études de réception, pour comprendre les multiples "anabases" vers l'antiquité, jusqu'à nos jours et me faire accepter par mes collègues « comparatistes ». J'emploie ce mot pour faire allusion à la revue Anabases fondée par Pascal Payen : j'ai connu Pascal à l'occasion de sa soutenance de thèse, à laquelle Nicole Loraux m'avait convié, peu de temps avant les jours dramatiques de ses accidents vasculaires célébraux (une autre soutenance, un peu plus tard, celle de Silvia Montiglio, à laquelle je participais aussi, dut être reportée en catastrophe pour cette raison); nous avons depuis lors développé des relations amicales et j'ai été très heureux quand il a pu intégrer l'université de Toulouse et y mener l'action remarquable que chacun connaît. Là se situe peut-être la seconde influence déterminante de "l'école de Paris » sur moi, qui rejoint une autre influence, celle de Jean Bollack : un philologue ne peut pas, je crois, se désintéresser de l'étude scientifique de l'histoire de la philologie, et, ajouterais-je, de la façon dont les non-philologues (et en particulier les grands écrivains) ont ou n'ont pas fait de place à l'antiquité dans la construction de leur univers de pensée, au sein de cette «culture générale », en réalité si diverse et complexe, dont parlait Vernant. 
10 Je suis ensuite revenu, comme professeur, à la Sorbonne (dans sa version appelée alors "Université Paris-IV Sorbonne, puis Paris-Sorbonne», et devenue maintenant "Sorbonne Université ") où j'ai assez rapidement assumé les fonctions de directeur de l'UFR de grec, puis de directeur de l'École doctorale «Mondes anciens et médiévaux ", jusqu'à ma retraite et à mon éméritat, en septembre 2018. L'histoire et la littérature grecques archaïques et classiques ne tiennent dans cette école doctorale qu'une place limitée et, dans la masse des doctorants (entre quarante et cinquante soutenances de thèse par an), bien peu, même parmi les classicistes, une infime minorité en fait, sont marqués par "l'école de Paris »; cela vaut aussi pour mes collègues, jeunes et moins jeunes, directeurs de thèse. Les travaux de plusieurs de ses membres sont mentionnés bien sûr, et connus, mais souvent plus par respect des principes bibliographiques que pour des raisons de fond. Réciproquement, j'ai pu constater dans plusieurs jurys de thèse que les travaux des philologues classiques sont souvent négligés, voire ignorés, par les doctorants de « l'école de Paris ». En poussant les choses un peu loin, je pourrais même dire que les travaux de cette "école » sont parfois plus connus et plus cités dans des thèses d'autres disciplines qui font intervenir l'antiquité et aux jurys desquels j'ai pu participer (philosophie, littérature «comparée »), que dans les thèses des classicistes : ils sont alors utilisés comme des autorités, comme s'ils dispensaient de revenir aux sources primaires et, d'une certaine façon, ils font ainsi partie désormais d'une nouvelle "culture générale universitaire », qu'il faut parfois déconstruire ou contester. En tout cas, la clôture entre classicistes à laquelle je faisais allusion plus haut est bel et bien, à mon avis, un risque réel. C'est en partie pour tenter de pallier ce risque qu'avec Glenn Most nous avons étendu à l'EHESS un séminaire doctoral annuel créé entre divers établissements pisans et parisiens (Scuola Normale Superiore, Università di Pisa, École normale supérieure de Paris, Sorbonne Université), avec l'appui de Riccardo Di Donato et de Claude Calame.

11 Il me faut pour finir évoquer un dernier aspect, qui fut conflictuel. Avec Jacqueline de Romilly, qui en prit si vigoureusement et si efficacement l'initiative, et d'autres, j'ai participé à la création de l'Association "Sauvegarde des enseignements littéraires ", qui a puissamment contribué à empêcher (ou peut-être seulement à retarder ?) la disparition du latin et du grec de l'enseignement du second degré en France, ces «langues mortes » si souvent condamnées comme élitistes et si souvent attaquées, par tous les gouvernements, pour des motifs divers. Nous voulions maintenir en France une partie de cette « culture générale » dont Vernant constatait la disparition à l'échelle du monde, ou du moins garantir aux collégiens et aux lycéens français de toutes origines la possibilité d'y avoir un peu accès, pour éviter une rupture, que nous estimions dommageable pour le lien civique et les rapports humains, avec tant d'éléments du passé culturel français et de la langue française. Ce combat s'est doublé d'un combat politique, car c'est un gouvernement de gauche qui a pris les initiatives les plus directes et les plus dangereuses. Jean-Pierre Vernant a eu le très grand courage d'y apporter son soutien et son autorité, même contre l'avis de certains de ses plus proches amis. Il a puissamment contribué à une certaine modification des idées reçues dans la gauche française, et en tout cas à l'abandon partiel de la réforme envisagée, quand Jack Lang remplaça Lionel Jospin au Ministère de l'Éducation Nationale. Je voudrais seulement témoigner ici pour finir de l'impact moral et intellectuel considérable, en particulier parmi les enseignants des lycées et collèges, du front commun qu'ils ont constitué tous les deux : la « tribu » en est sortie provisoirement ragaillardie et renforcée. 


\section{RÉSUMÉS}

Témoignage d'un "Sorbonnard" sur ses contacts avec Vernant et les siens au cours de sa carrière d'helléniste.

How a teacher and professor of ancient Greek language and literature at Sorbonne University met Vernant and his fellows : a testimony.

INDEX

Mots-clés : histoire des idées, structuralisme, philologie, narratologie, Sorbonne

Keywords : history of ideas, structuralism, philology, narratology, Sorbonne

\section{AUTEUR}

\section{PAUL DEMONT}

Sorbonne Université, Orient \& Méditerranée UMR 8176 